\title{
A Proposed Mechanism for the Initial Myosin Binding Event on the Cardiac Thin Filament: a Metadynamics Study
}

Anthony P. Baldo, ${ }^{+}$Jil C. Tardiff, " and Steven D. Schwartz ${ }^{*}+$

†Department of Chemistry and Biochemistry, The University of Arizona, Tucson, AZ 85721

ףDepartment of Biomedical Engineering, The University of Arizona, Tucson, AZ 85724

*Corresponding Author

E-mail:sschwartz@email.arizona.edu 


\section{Contents}

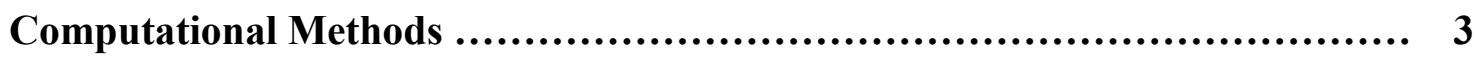

Tm Movement Discussion $\ldots . \ldots \ldots \ldots \ldots \ldots \ldots \ldots \ldots \ldots \ldots \ldots \ldots \ldots \ldots \ldots \ldots \ldots \ldots \ldots \ldots \ldots . \ldots \ldots$

Figure $S 1$.................................................................. 10

Figure $\mathbf{S 2}$................................................................. 12

Figure $\mathbf{S 3}$.................................................................. 14

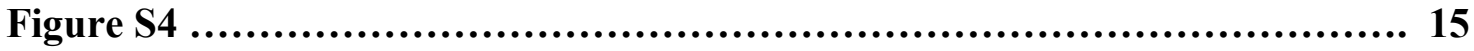

Table $\mathbf{S 1}$................................................................... 16

Table $S 1$............................................................................ 16

Figure $S 5$ [....................................................................... 17

Supplemental References .................................................... 18 


\section{Computational Methods}

The structure utilized in this study were an update to our previous atomistic structure of the CTF.(1) Yamada, Namba, and Fujii published a seminal CEM study that revealed a new orientation of the troponin complex within the thin filament(2) as well as demonstrating calcium depleted (Ca-) and saturated $(\mathrm{Ca}+)$ thin filament structures. One of the main differences from previous studies is the orientation of cTnT within the overlap region of the thin filament, with this new orientation also being previously observed computationally in a protein docking study by Pavadai et al.,(3) though we note there is a slight difference in the placement of the Nterminal end of TnT between these two models. Additional protein-protein docking was also performed by Pavadai et al. to orient tropomyosin on actin as it was observed that the Tm chains in the Yamada structure were over compressed and does not represent the canonical structure of Tm,(4) though the study showed good agreement with the relative positions of Tm on the actin in the $\mathrm{Ca}+$ state.

To define the initial and final position of tropomyosin from the myosin binding event, we updated our atomistic structure of the CTF to explicitly include myosin bound in the appropriate positions as well as update the orientation of the troponin complex as resolved with recent structural studies.(2,3) Therefore, information on tropomyosin's myosin-bound, M-state, position with respect to actin as well as a structure for human cardiac myosin was required. Protein database entries $4 A 7 H(5)$ and $5 H 53(6)$ provided the reference coordinates for tropomyosin and myosin respectively. We note a higher resolution structure of an actin-tropomyosin-myosin (ATM) complex has been published by Von Der Ecken et al.,(6) but the structures differ little in 
tropomyosin's position on the actin surface. More recently, additional information of the ATM complex with cardiac myosin has been presented by Doran et al.(7) as well as Risi et al.,(8) but were unavailable at the time for the preparation of the metadynamics simulations due to the extensive computational hours required. However, since a ATM structure was only needed to define the M-state of Tm, of which they are all in general agreement, we expect the influence of these new structures on the observations to be minimal.

However before a M-state model of tropomyosin was developed, we updated our previous model to reorient the troponin core to correspond to recent CEM images of the thin filament as well as a computational docking study.(9,10) PDB $6 K N 7$ and PDB $6 K N 8$ represent the thin filament in $\mathrm{Ca}$ - and $\mathrm{Ca}+$ states respectively as determined by Yamada, Namba, and Fujii.(10) These served as a template through which we would modify our previous atomic model to match in order to create two structures, calcium depleted and abundant, while still retaining portions of the individual protein chains unresolved by Yamada, Namba, and Fujii. Coordinates for the thin filament from PDB $6 K N 7$ and PDB $6 K N 8$ were aligned with our previous model by minimizing the root mean squared displacement (RMSD) between the backbone atoms of the actin monomers, using the overlap region of the thin filament as a reference point. From there, the Tn proteins were translated/rotated as a rigid body from our previous model to minimize the RMSD with their Yamada et al. counterparts. Further refinement was achieved for each individual protein chain by running dynamics using the internal coordinates from Yamada et al. as constraints. Each Tn chain was subject to minimization, heating, and equilibration with the aforementioned constraints with the NanoScale Molecular Dynamics (NAMD)(11) package version 2.12 with the CHARMM36(12) force field. In each 
case for Tn refinement, implicit solvent was utilized with the Generalized Born (GB) scheme with a solvent dielectric constant value of 80 and a set ion concentration of $0.15 \mathrm{M}$.

Also, Tm's position on the actin surface needed to be updated to reflect the positions examined by Yamada et al. However, as discussed previously, the Tm chains in Yamada et al. were overcompressed ${ }^{4}$ and does not represent the canonical structure of $\mathrm{Tm}$, therefore their atomic coordinates could not be utilized explicitly. In that case, the Tm dimers from our previous model were translated as a rigid body perpendicular with respect to the actin filament until its position reflected that observed by Yamada et al.,(2) utilizing the overlap region of the thin filament as a point of reference while retaining the same helical pitch and orientation observed by $\mathrm{Li}$ et al.(13) Dynamics were run on the actin-tropomyosin complex in its refined position in a similar manner as previously mentioned. Then the appropriate fragments were compiled to create a calcium deleted structure, with Tm in a blocked position and is constrained there from interactions originating in the Tn complex as in PDB 6KN7. An additional structure of the thin filament was created with Tm in its blocked position, but the Tn complex structure is as in PDB $6 K N 8$ and does not constrain $\mathrm{Tm}$. This system was the input structure for the metadynamics $(14,15)$ simulations and will be referred to as the hybrid structure. Figure S1 shows that the refined placement of the canonical Tm chain in the blocked state on the actin surface fits quite well in the EM density of Yamada et al.(2) without its explicit use, which was of low resolution.(4)

Additionally, a M-state structure of the thin filament had to be created as a target for the metadynamics simulations and therefore, the Tm M-state had to be defined. Initially to translate topomyosin to the M-state from its open position, the atomic coordinates from PDB $4 A 7 H(5)$ were utilized. This structure contains Dictyostelium myosin in its rigor state bound to actin and 
tropomyosin, but only the atomic coordinates for Tm were used. The program Visual Molecular Dynamics (VMD)(16) version 1.9.3 was used to align PDB $4 A 7 H$ with our hybrid model of the CTF by minimizing the RMSD between the backbone atoms of the actin monomers on $4 A 7 H$ with those in our model. Afterwards tropomyosin was translated as a rigid body (no change in internal coordinates or rotation about tropomyosin's major axis) until its backbone atom coordinates were $<2 \AA$ from the reference coordinates of the tropomyosin segment in PDB $4 A 7 H$. The whole system was then solvated using the SOLVATE plugin in VMD 1.9.3 with TIP3 waters extending at least $15 \AA$ from the protein surface. Counter ions of potassium and chloride ions were added to the solvated box with the AUTOIONIZE plugin in VMD 1.9.3 to a set concentration of $0.15 \mathrm{~mol} / \mathrm{L}$.

The prepared structure was then utilized as input for MD simulations with the NAMD package version 2.12 with the CHARMM36 force field. The complete system was initially subject to minimization and heating-equilibration at $70 \mathrm{~K}$ to relax residue-residue contacts between tropomyosin and actin. 5000 steps of minimization were performed with the conjugate gradient method and then heated at a rate of $1 \mathrm{~K} / \mathrm{ps}$ to the desired temperature. Finally, an equilibration run of $1 \mathrm{~ns}$ was generated in an isobaric, isothermal ensemble (1 atm, $70 \mathrm{~K})$. Afterwards, further refinement of the tropomyosin chain over the actin surface was required to reflect PDB $4 A 7 H$. To perform this, harmonic constraints were enforced to the backbone atoms of tropomyosin with reference coordinates being provided from the aligned structure of PDB $4 A 7 H$. This led to an additional $1 \mathrm{~ns}$ equilibration run with these constraints. The final structure of the constrained equilibrium was then subject to minimization and heating to $300 \mathrm{~K}$ in a similar scheme as previously described with a $10 \mathrm{kcal} / \mathrm{mol}$ harmonic force constant on the tropomyosin chain to prevent any deviation from its refined $\mathrm{M}$ position. Equilibration runs of $1 \mathrm{~ns}$ each were 
then run with decreasing constraints, with force constants of 10, 7, 5, 3, 1, and $0 \mathrm{kcal}$, on the tropomyosin backbone. The final structure of the unconstrained equilibration reflected the CTF with tropomyosin in its apo, M-state (no myosin present). Figure S2 compares our M-state position of Tm with that determined by Doran et al.(7)

The homology model for myosin was created with the SWISSMODEL(17) program utilizing PDB 5H53(6) as the template and the sequence of $M Y H 7$ from Uniprot entry P12883. The outputted structure of human beta myosin (residues 1 to 800) as compared to the rabbit skeletal template is shown in Figure S3. Having the myosin structure and Tm-M state structure prepared, we initially aligned PDB 5H53 with the equilibrated M-state CTF by means of their respective actin monomers and then aligned the human myosin structure with the rabbit skeletal myosin according to their backbone atoms. The new coordinates for the human myosin-CTF structure were then subject to minimization, heating, and equilibration as previously stated at 70 $\mathrm{K}$ and $300 \mathrm{~K}$ with decreasing constraints $(10,7,5,3,1,0)$ on myosin backbone atoms enforced during the initial minimization and heating $70 \mathrm{~K}$ runs to relax residue contacts. This docking procedure was repeated to prepare one structure with myosin bound in the linker region of the $\mathrm{CTF}$ and another structure with myosin in the overlap region. This myosin-CTF structure was utilized to confirm the refined M-state of Tm. Figure S4 shows that these rigorous computational refinements were able to resolve the important contacts between loop 4 of myosin and tropomyosin recently highlighted by Doran et al.(7) and Risi et al.(8) Additionally, tables S1-S2 and Figures S5 provide a summary of the utilized structures and a general workflow for system preparation. 
Metadynamics $(14,15)$ simulations of linker and overlap positions began from the previously mentioned hybrid structure without myosin explicitly bound. The atomic coordinates for the CTF were subject to minimization, heating, and equilibration as previously described. The collective variable chosen to distinguish our initial and final positions is the center of mass distance between a selected group of alpha carbons from Tm in the initial structure and the respective final structures for the linker (residues 180 to 220), overlap (Cterm: residues 266 to 284; Nterm: residues 1-35), and cooperative (residues 225 to 260) simulations.

For the cooperative mechanism, a conformation from the linker metadynamics simulation when the tropomyosin chain was in its M-state was taken and myosin was explicitly docked in that position to hold tropomyosin in place. From that state, another metadynamics simulation was performed in which the next immediate segment in the Tm chain was biased to its M-state. A constraining force of $5 \mathrm{kcal} / \mathrm{mol}$ was also imposed on the alpha carbons of the actin monomers to prevent an undesirable deformation of the thin filament due to the metadynamics bias. Convergence of metadynamics simulations was determined when additional sampling (i.e. running the simulation longer) resulted in little to no change in the observed FEP and/or the lowest free energy difference estimate was achieved. Sampling was performed up to $0.4 \AA$ from the M-state to prevent interference of the PMF calculation with external constraints enforced to keep sampling within the desired region.

Gaussian biases were deposited in the simulation every 2 ps of $0.5 \mathrm{kcal} / \mathrm{mol}$ in height, of which were utilized to determine the underlying FEPs. Small biases as well as long deposition rates minimize non-equilibrium effects while adversely affecting computational times required. All MD simulations were performed in NAMD 2.12 with CHARMM36 parameters. Collective 
variable definition and metadynamics simulations were defined and utilized with the aid of the Collective Variable Module(18) as implemented in NAMD.

\section{Tm Movement Discussion}

One of the significant observations made by Yamada et al.(2) was that for Tm movement, the chains seemed to roll between the calcium depleted and calcium abundant states of the thin filament to reach the blocked and close states respectively. Additionally, the amount of rolling was dependent on the relative position of each segment of Tm within the thin filament. They observed little to no rolling in the overlap, where one Tm dimer interacts with another, while a more significant effect is observed towards the $\mathrm{C}$-terminal end of the chain. A recent review by Tobacman provides additional description to this type of rotation described by Yamada et al.(2) between the blocked and closed states, with Pavadai et al. making a similar observation. So, the question remains, does tropomyosin roll or slide across the actin surface during thin filament activation?

In the metadynamics simulations presented here, the small segments of Tm biased travel azimuthally with respect to the major axis of the actin filament, as noted previously.(19) The estimated amount of Tm rolling that occurred in the overlap and linker simulation for the biased segments were $\sim 11$ degrees and $\sim 25$ degrees respectively. However, during the linker simulation the magnitude of Tm rolling was at times observed to be as large as $\sim 50$ degrees, which is on the order of Tm rolling between the blocked and closed states observed by Pavadai et al. (4) This suggests a degree of Tm rolling does occur, however the severity of the rolling is restricted in the metadynamics simulations as only $14 \%$ of the Tm chain is transitioning while 
the remaining $86 \%$ remains near the blocked state. As further myosin binding occurs, the degree of Tm rolling might become more pronounced.

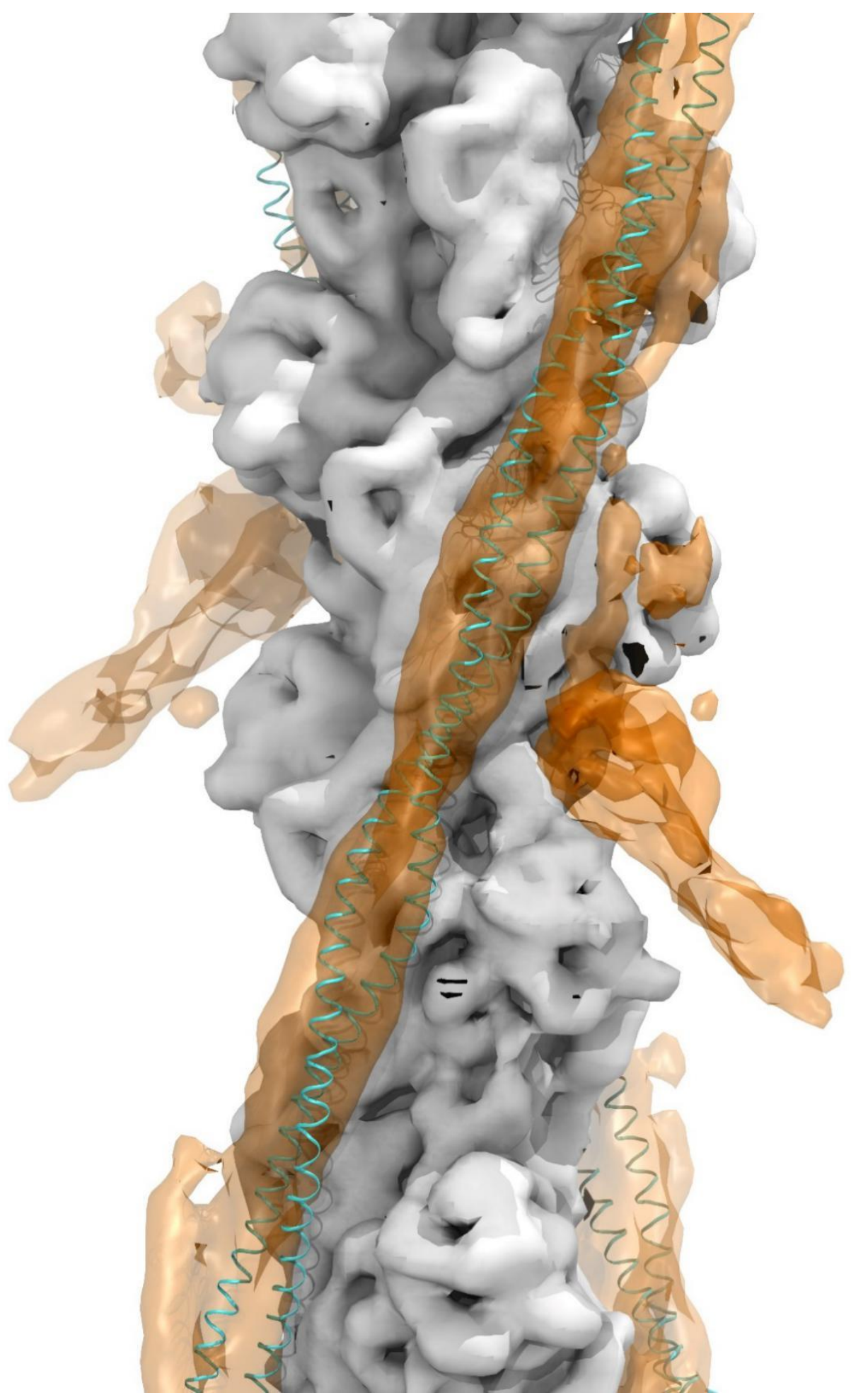


Figure S1. Atomic tropomyosin (blue) in the blocked state overlayed with the EM density (orange/gray) from Yamada et al.(2) Atomic troponin not shown for clarity. Discrepancies between the atomic model and EM density are within thermal fluctuations. 

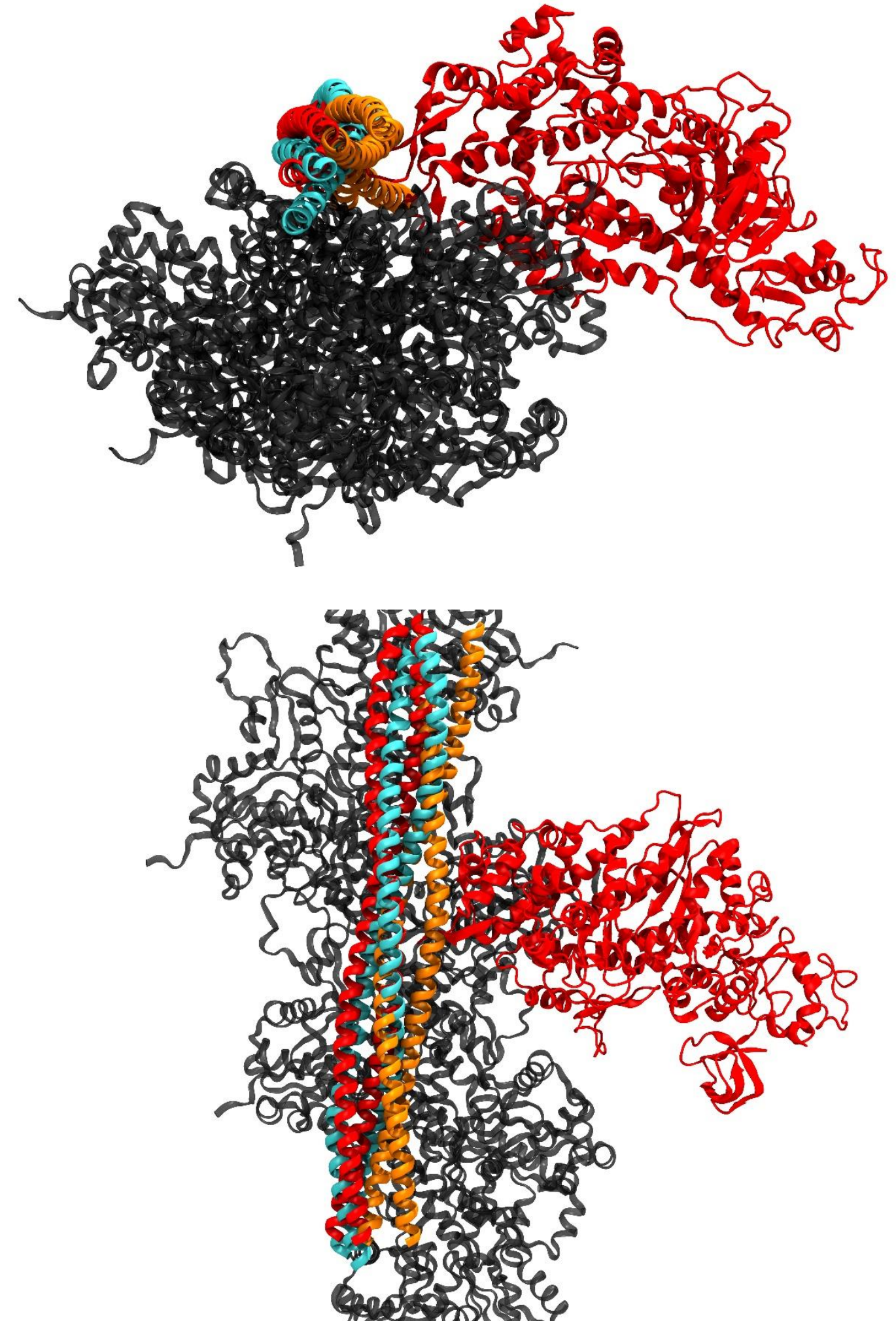
Figure S2. Side (upper) and top (lower) views of Actin (gray), tropomyosin (red; residues 45 to 140), and myosin (red) structure as determined by Doran et al.(7) compared to Tm in the closed state (orange) determined by Pavadai et al.(4) and the M-state of Tm (blue; a given conformation from the equilibration process) utilized in this study. Troponin not shown for clarity. Normalized projections were calculated between the difference vectors of two residue pairs (76/77 and 117/118) in our M-state and the Doran M-state. The observed projections were 0.98 and 0.96 respectively, denoting a similar orientation of the Tm chains with respect to the actin surface between the two M-state models. Additional differences are within thermal fluctuations. 


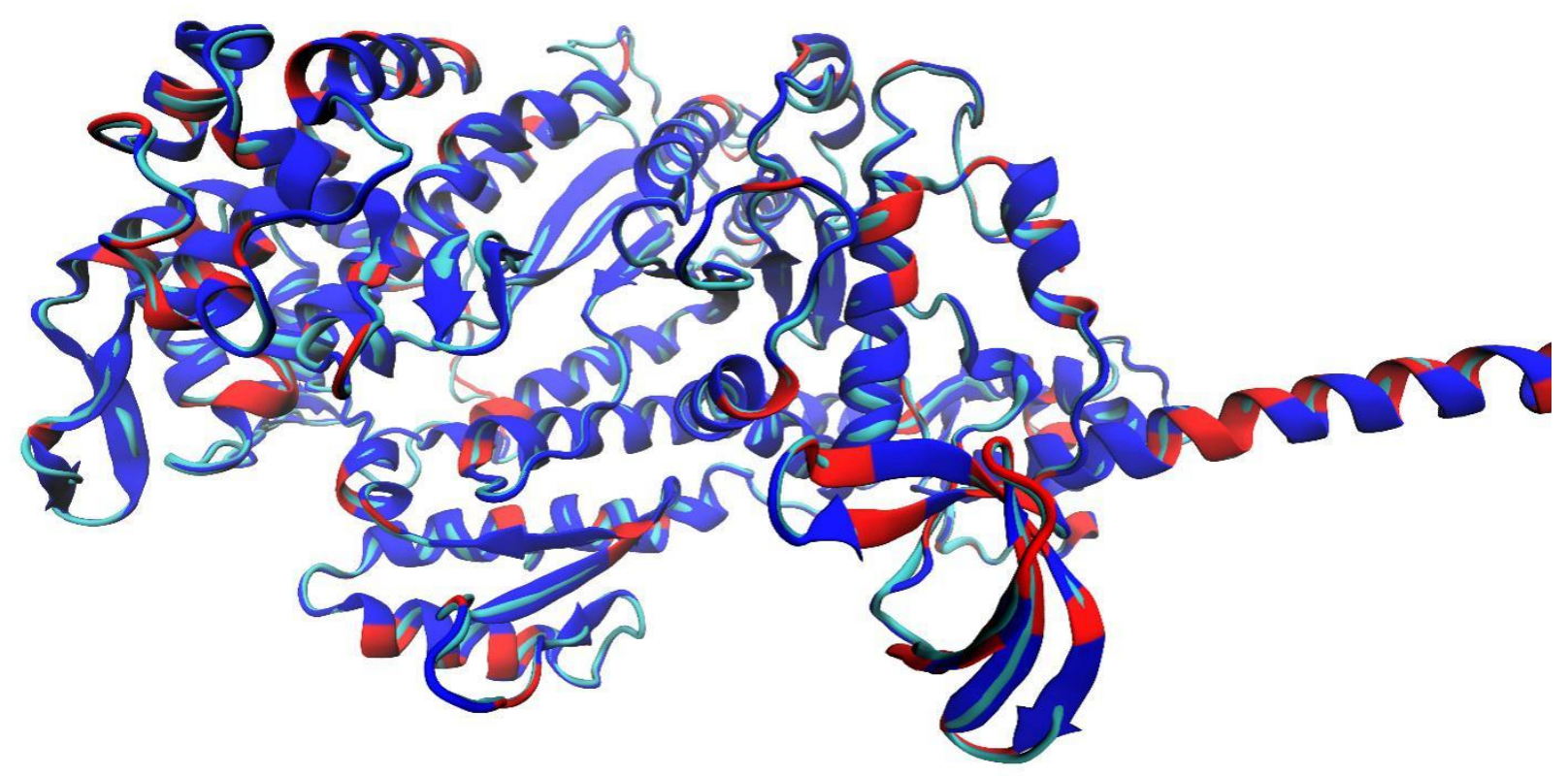

Figure S3. Homology model of human, cardiac myosin ( $\beta$ isoform), as shown in blue/red as compared to the rabbit skeletal template (cyan). The blue and red regions highlight similar and dissimilar residues between the two myosins respectively. 


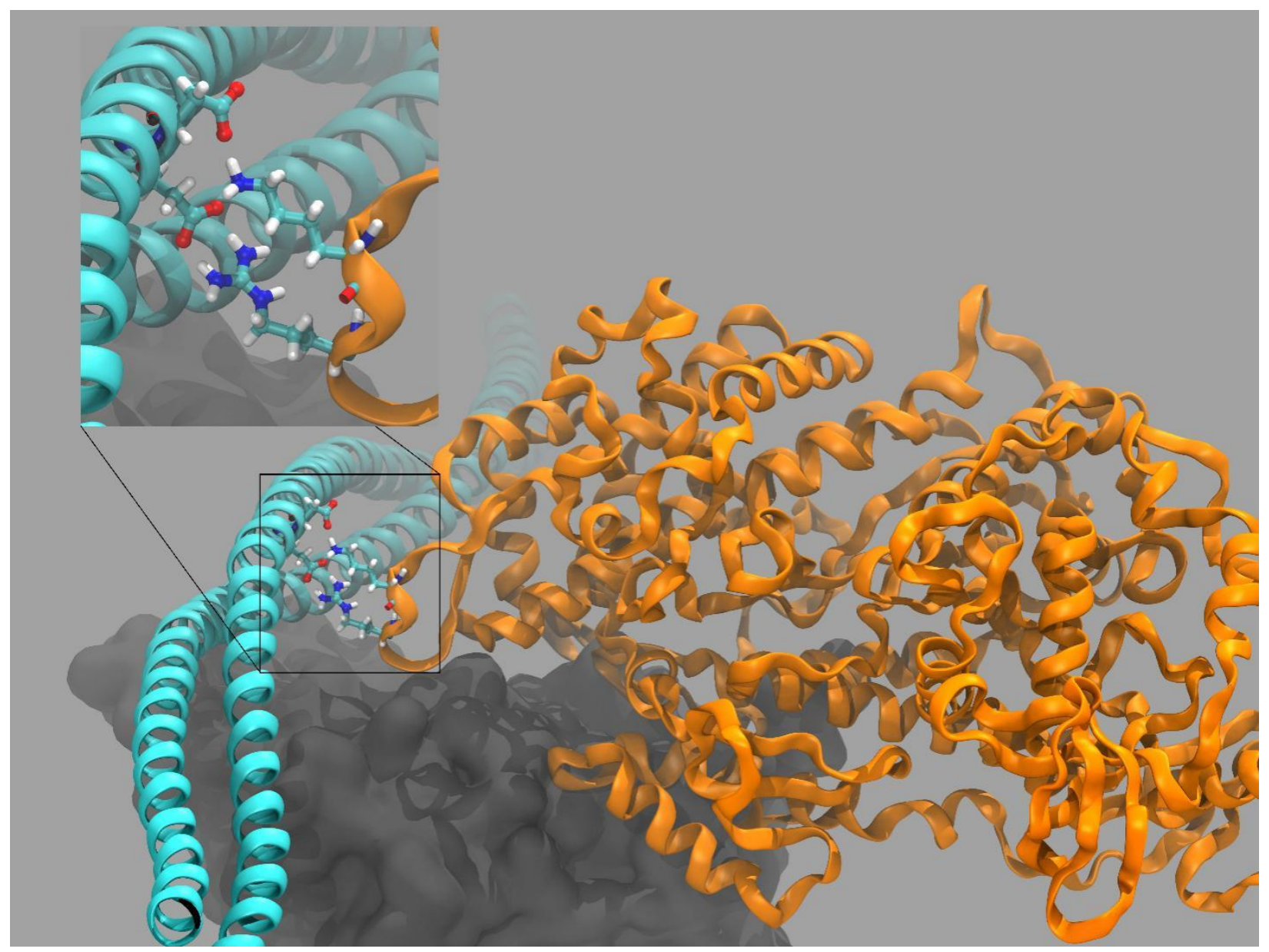

Figure S4. The myosin-tropomyosin interface. Residues Lys367 and Arg369 extend from loop 4 of cardiac myosin to contact acidic residues (Glu218 and Asp219) on tropomyosin. Doran et al.(7) observed Arg369 and Glu218 coming within $5 \AA$ of each other in both their docking and molecular dynamics studies. 
Table S1: Summary of the starting structures and those used to refine them in the generation of the hydrid CTF structure, which used as the input for the metadynamics simulations.

\begin{tabular}{c|c|c|c|c} 
& Tm + actin & TnT & TnI & TnC \\
\hline Starting Structures & Williams model(1) & Williams model & Williams model & Williams model \\
\hline Refinement Structures & PDB 6KN7(2) & PDB 6KN8(2) & PDB 6KN8 & PDB 6KN8
\end{tabular}

Table S2: Summary of the starting structures and those used to refine them in the generation of the myosin bound CTF structure, which was used to define the collective variable for the metadynamics simulations. *Homology modeling was utilized to generate the human, $\beta$ myosin structure with the rabbit skeletal myosin from PDB $5 H 53$ serving as the template.

\begin{tabular}{c|c|c|c|c|c} 
& $\mathrm{Tm}+$ actin & $\mathrm{TnT}$ & $\mathrm{TnI}$ & $\mathrm{TnC}$ & Nyosin \\
\hline Starting Structures & Williams model(1) & Williams model & Williams model & Williams model & PDB 5H59.9(6) \\
\hline Refinement Structures & PDB 4A7H(5) & PDB 6KN8(2) & PDB 6KN8 & PDB 6KN8 & $n / \mathrm{a}^{*}$
\end{tabular}



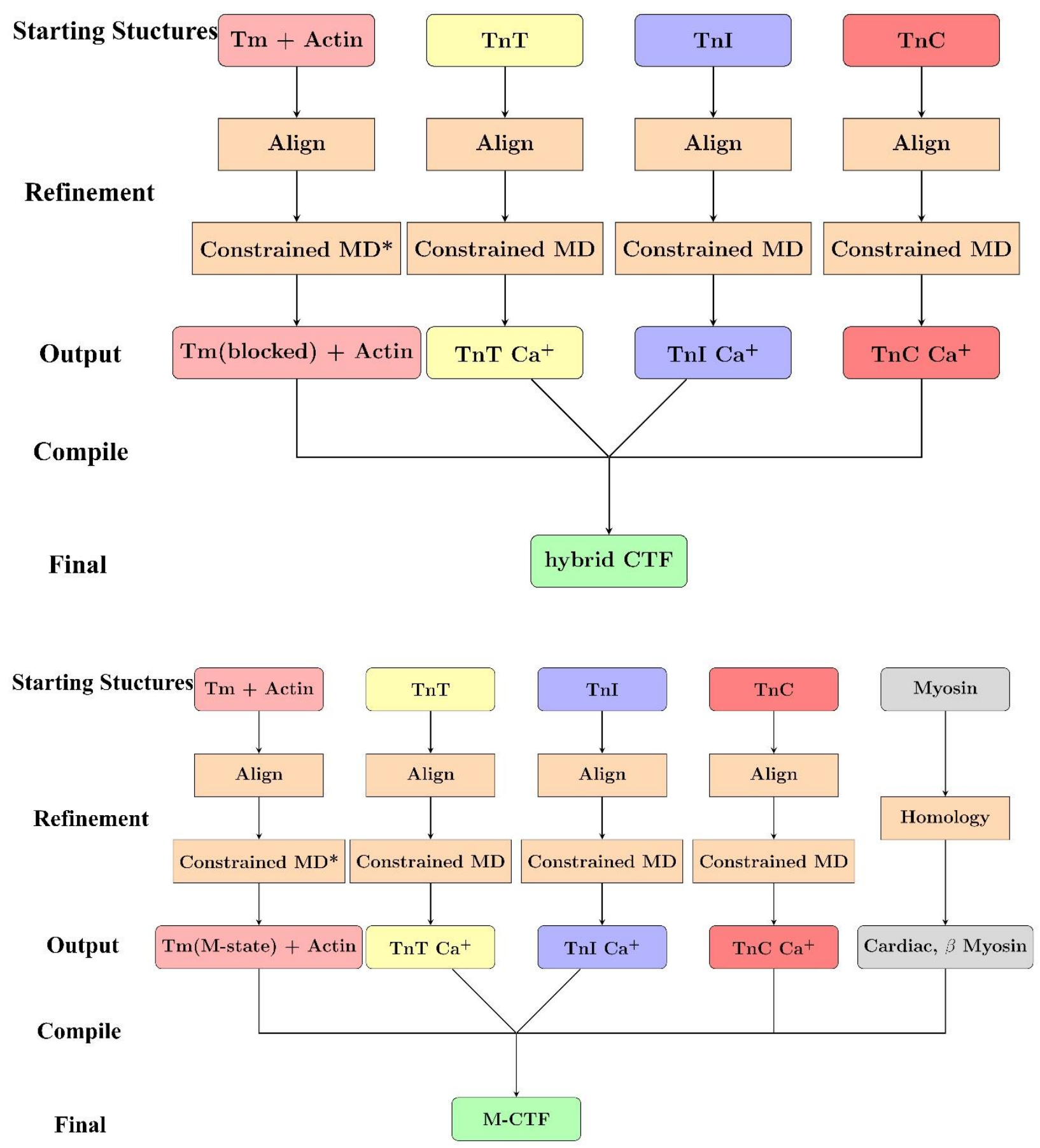

Figure S5: General workflow for producing the structures utilized in this structure as the input (top) of the metadynamics and target (bottom) used to define the collective variable with the structures utilized summarized in tables S1-S2. Starting structures are aligned to their 
refinement structures and MD runs were performed using the atomic coordinates from the refinement structures as constraints. *For Tm chain, the atomic coordinates were not used explicitly and constraints were applied according to center of masses.

\section{References}

(1) Williams, M. R.; Lehman, S. J.; Tardiff, J. C.; Schwartz, S. D. Atomic Resolution Probe for Allostery in the Regulatory Thin Filament. Proc. Natl. Acad. Sci. U. S. A. 2016, 113, 3257-3262.

(2) Yamada, Y.; Namba, K.; Fujii, T. Cardiac Muscle Thin Filament Structures Reveal Calcium Regulatory Mechanism. Nat. Commun. 2020, 11, 1-9.

(3) Pavadai, E.; Rynkiewicz, M. J.; Ghosh, A.; Lehman, W. Docking Troponin T onto the Tropomyosin Overlapping Domain of Thin Filaments. Biophys. J. 2020, 118, 325-336.

(4) Pavadai, E.; Lehman, W.; Rynkiewicz, M. J. Protein-Protein Docking Reveals Dynamic Interactions of Tropomyosin on Actin Filaments. Biophys. J. 2020, 119, 75-86.

(5) Behrmann, E.; Müller, M.; Penczek, P. A.; Mannherz, H. G.; Manstein, D. J.; Raunser, S. Structure of the Rigor Actin-Tropomyosin-Myosin Complex. Cell 2012, 150, 327-338.

(6) Fujii, T.; Namba, K. Structure of Actomyosin Rigour Complex at $5.2 \AA$ Resolution and Insights into the ATPase Cycle Mechanism. Nat. Commun. 2017, 8, 13969.

(7) Doran, M. H.; Pavadai, E.; Rynkiewicz, M. J.; Walklate, J.; Bullitt, E.; Moore, J. R.; Regnier, M.; Geeves, M. A.; Lehman, W. Cryo-EM and Molecular Docking Shows Myosin Loop 4 Contacts Actin and Tropomyosin on Thin Filaments. Biophys. J. 2020, 119, 821-830.

(8) Risi, C.; Schäfer, L. U.; Belknap, B.; Pepper, I.; White, H. D.; Schröder, G. F.; Galkin, V. E. HighResolution Cryo-EM Structure of the Cardiac Actomyosin Complex. Structure 2021, 29, 50-60.

(9) Pavadai, E.; Rynkiewicz, M. J.; Ghosh, A.; Lehman, W. Docking Troponin T onto the Tropomyosin Overlapping Domain of Thin Filaments. Biophys. J. 2020, 118, 325-336.

(10) Yamada, Y.; Namba, K.; Fujii, T. Cardiac Muscle Thin Filament Structures Reveal Calcium Regulatory Mechanism. Nat. Commun. 2020, 11, 153

(11) Phillips, J. C.; Braun, R.; Wang, W.; Gumbart, J.; Tajkhorshid, E.; Villa, E.; Chipot, C.; Skeel, R. D.; Kalé, L.; Schulten, K. Scalable Molecular Dynamics with NAMD. J. Comp. Chem. 2005, 1781-1802.

(12) Best, R. B.; Zhu, X.; Shim, J.; Lopes, P. E. M.; Mittal, J.; Feig, M.; MacKerell, A. D. Optimization of the Additive CHARMM All-Atom Protein Force Field Targeting Improved Sampling of the Backbone $\phi, \psi$ and Side-Chain X1 and X2 Dihedral Angles. J. Chem. Theory Comput. 2012, 8, 3257-3273.

(13) Li, X. E.; Orzechowski, M.; Lehman, W.; Fischer, S. Structure and Flexibility of the Tropomyosin Overlap Junction. Biochem. Biophys. Res. Commun. 2014, 446, 304-308.

(14) Laio, A.; Parrinello, M. Escaping Free-Energy Minima. Proc. Natl. Acad. Sci. U. S. A. 2002, 99, 
$12562-12566$.

(15) Laio, A.; Gervasio, F. L. Metadynamics: A Method to Simulate Rare Events and Reconstruct the Free Energy in Biophysics, Chemistry and Material Science. Reports Prog. Phys. 2008, 71, 126601.

(16) Humphrey, W.; Dalke, A.; Schulten, K. VMD: Visual Molecular Dynamics. J. Mol. Graph. 1996, 14, 33-38.

(17) Schwede, T.; Kopp, J.; Guex, N.; Peitsch, M. C. SWISS-MODEL: An Automated Protein HomologyModeling Server. Nucleic Acids Res. 2003, 31, 3381-3385.

(18) Fiorin, G.; Klein, M. L.; Hénin, J. Using Collective Variables to Drive Molecular Dynamics Simulations. Mol. Phys. 2013, 111, 3345-3362.

(19) Williams, M. R.; Tardiff, J. C.; Schwartz, S. D. Mechanism of Cardiac Tropomyosin Transitions on Filamentous Actin As Revealed by All-Atom Steered Molecular Dynamics Simulations. J. Phys. Chem. Lett. 2018, 9, 3301-3306. 